\title{
A Compact Wide-band EBG Structure Utilizing Embedded Resonant Circuits
}

\author{
Hossein Mosallaei, Senior Member, IEEE, and Kamal Sarabandi, Fellow, IEEE
}

\begin{abstract}
This letter presents a wide-band electromagnetic bandgap (EBG) structure designed utilizing embedded resonant circuits (ERCs). The EBG structure is a periodic material whose unit cell is composed of a number of small resonant $L C$ circuits. For EBG composed of single resonant $L C$ circuits above the resonant frequency, the structure behaves as a negative permeability material that provides the bandgap behavior. To design a compact wide-band EBG, a structure constructed of three layers of ERC, each having different resonant frequencies separated by layers of impedance inverters, is proposed. The impedance inverter layer is designed using $I$-shaped metallic inclusions printed in the host medium to achieve a high dielectric material so as to physically reduce the thickness of the quarter wavelength inverters. The design and performance characteristic of a complete EBG structure with isotropic bandgap behavior almost independent of incident angle and polarization state is also demonstrated.
\end{abstract}

Index Terms-Electromagnetic bandgap (EBG), embedded-circuit, finite difference time domain (FDTD), miniaturization, negative permeability, periodic structure, wideband.

\section{INTRODUCTION}

$\mathbf{E}$ LECTROMAGNETIC bandgap (EBG) materials have a wide range of applications in RF and microwave engineering including microwave and optical cavities, filters, waveguides, and smart artificial surfaces, etc. [1]-[8]. Traditionally, bandgap behavior is achieved using a periodic dielectric or metallic structure with periodicity value comparable to the wavelength. Usually, 4-5 periods are needed to provide good bandgap characteristics (high isolation), and therefore, a large physical space is required for integrating the EBG into a system.

For most RF applications compact size is a requirement, and therefore, it is very desirable to obtain a compact EBG structure with high isolation. To accomplish this, we propose a bandgap structure based on an embedded-circuit meta-material composed of tank circuits arranged in a periodic fashion with a periodicity much smaller than a wavelength. As will be shown, a thin layer of such a medium containing even one layer of tank circuits can provide significant isolation.

Transmission line theory developed in [9] is applied to obtain an equivalent circuit model for the medium and conceptually investigate the performance of embedded-circuits. An equivalent medium with $\varepsilon_{\text {eff }}-\mu_{\text {eff }}$ parameters for the complex periodic structure is determined. It is shown that the embedded-circuit

Manuscript received August 27, 2004; revised November 10, 2004.

The authors are with the Electrical Engineering and Computer Science Department, University of Michigan, Ann Arbor, MI 48109-2122 USA (e-mail: hosseinm@engin.umich.edu).

Digital Object Identifier 10.1109/LAWP.2004.841213 medium has a constant effective permittivity and a frequency dependent effective permeability. Above the resonant frequency, where the permeability is negative, the wave cannot propagate through the medium and a bandgap characteristic is observed. By tailoring the circuit inclusions and their geometry the performance of the EBG can be controlled. Cascading embedded-circuit layers with different resonant frequencies interleaved with layers of impedance inverters allows for achieving a multiresonant wide-band behavior. A compact three-layered EBG material is successfully designed to provide a wide bandgap behavior having a minimum of $20-\mathrm{dB}$ isolation over $25 \%$ bandwidth.

The analytical results evaluated based on the transmission line theory are validated by a finite-difference time-domain (FDTD) full-wave approach. The FDTD code used in this investigation is very versatile and allows for placement of the periodic boundary conditions/perfectly matched layers (PBC/PML) walls in designed locations according to the problem geometry, wave polarization, and direction of propagation [10]-[12].

\section{EMBEDDED RESONANT CIRCUITS}

The earliest work regarding the design of artificial magnetism using resonant circuit inclusions dates back to 1952, when the subject was investigated by Schelkunoff et al. [13]. The same concept was further developed by Pendry et al. in 1999 [14], utilizing split ring resonators (SRRs). A general formulation based on the transmission line theory, applicable for analysis and synthesis of complex materials composed of dielectric, magnetic, and metallic inclusions, has recently been reported in [9]. Here, we address the physical concept of a novel EBG material designed utilizing ERC, based on the techniques presented in [9].

Fig. 1(a) depicts the geometry of a periodic structure of loop circuits embedded in a low dielectric host medium. The loops are terminated to the loading capacitors $C_{p}$. A building block unit cell of the medium is shown in Fig. 1(b). For a plane wave, propagating in a normal direction with $z$-polarized electric field and $y$-polarized magnetic field, one can terminate the unit cell with PEC and PMC walls as illustrate in Fig. 1(b).

The transmission line method is applied to obtain a circuit model for the unit cell of the structure [Fig. 1(c)] and determine the physical parameters of the ERC medium. The current on the transmission line generates a magnetic flux passing through the loops in $y$-direction that it induces a current in the loops. The loops in $y$-direction can be viewed as a toroid with inductance $L_{p}$ perturbing the magnetic property of the host medium. Also, there is a coupling capacitance $C_{c}$ between the loops in the vertical direction ( $z$-direction) that artificially changes the permittivity of the host medium. It is determined in [9] that the 


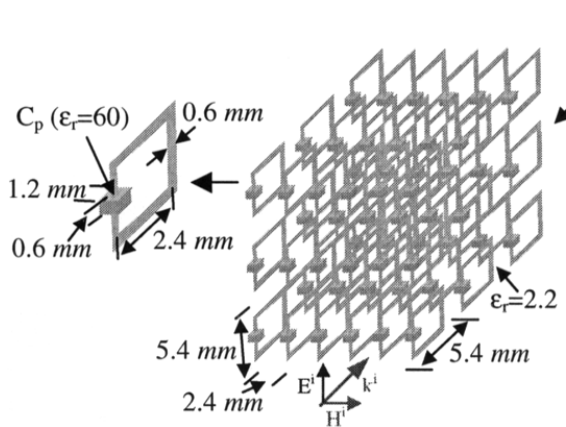

(a)

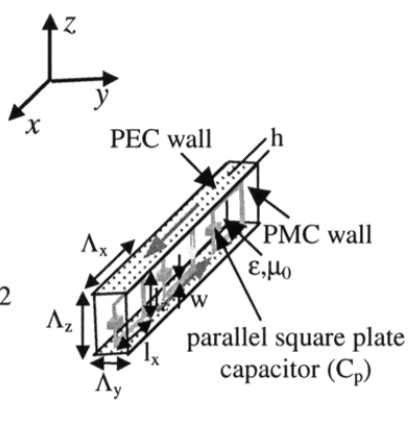

(b)

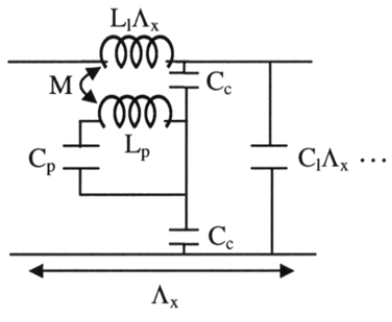

(c)

Fig. 1. Single resonant three-layered ERC EBG material. (a) Geometry of the structure with periodicity in the $y-z$ directions. (b) Building block unit cell terminated to the PEC walls in the $x y$-planes and PMC walls in the $x z$-planes. (c) Equivalent circuit model. Note that a building block of the structure has a very small size.

periodic ERC is equivalent to a homogeneous medium with the following constitutive parameters:

$$
\begin{aligned}
& \varepsilon_{\mathrm{eff}}=\varepsilon\left[1+\frac{\Lambda_{z}\left(l_{x}+w\right)}{\Lambda_{x} \Lambda_{y}} \frac{K\left(\sqrt{1-g^{2}}\right)}{K(g)}\right] \\
& \mu_{\mathrm{eff}}=\mu_{0}\left(1-\kappa^{2} \frac{1}{1-\frac{\omega_{p}^{2}}{\omega^{2}}}\right)
\end{aligned}
$$

where

$$
\begin{array}{cc}
g=\frac{h}{(h+w)} \quad \kappa^{2}=\frac{\left[\left(l_{x}-w\right)\left(l_{z}-w\right)\right]}{\Lambda_{x} \Lambda_{z}} \\
\omega_{p}=\frac{1}{\sqrt{L_{p} C_{p}}} \quad L_{p}=\frac{\mu_{0}\left(l_{x}-w\right)\left(l_{z}-w\right)}{\Lambda_{y}}
\end{array}
$$

and $K$ is the complete elliptic integral function. It is shown that ERC medium presents a constant effective permittivity which is higher than the dielectric constant of the host medium. On the other hand the effective permeability of the medium shows a resonant behavior. Below the resonance, $\mu_{\text {eff }}$ is positive, and the wave can propagate through the structure. However, above the resonance, where $\mu_{\mathrm{eff}}$ is negative, the wave cannot propagate through the medium or a bandgap phenomenon is observed.

An FDTD with PBC/PML walls is applied to numerically characterize the complex periodic ERC designed in Fig. 1(a). A plane wave illumination is used to calculate the transmission coefficient at normal incidence, and the result is shown in Fig. 2. As predicted by the transmission line model above the resonance, a stopband or bandgap behavior is illustrated. Tailoring the loop configuration allows one to easily control the bandgap response. One can lower the resonant frequency of the ERC EBG by simply using a larger loop capacitor but keeping the same loop size. This means that by increasing the loop capacitor the size of the unit cell can be successfully reduced. The loops in this example have a side length smaller than $0.05 \lambda_{0}$.

\section{WIDEBAND BANDGAP MATERIAL USING A MULTIRESONANT STRUCTURE}

In the previous section, an ERC bandgap structure with a miniaturized size building block unit cell was presented. One limitation of this design is that the bandwidth of the stop-band

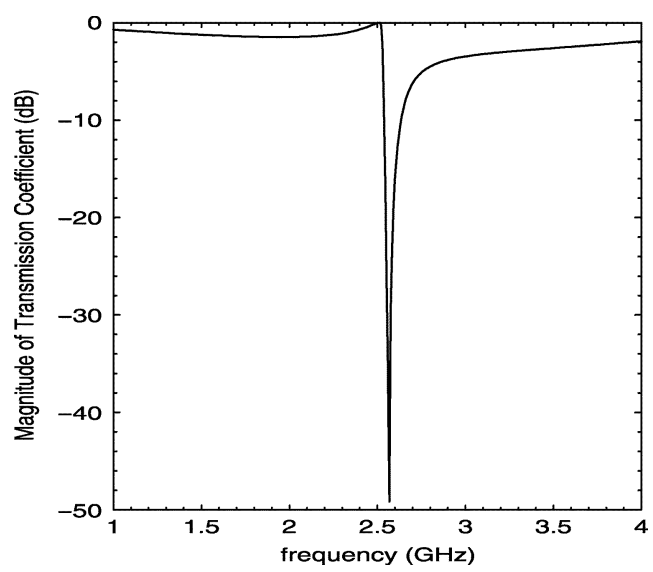

Fig. 2. Transmission coefficient for a plane wave propagating through the single resonant EBG medium designed in Fig. 1(a). The negative value of $\mu_{\text {eff }}$ above its resonant frequency produces the stop-band performance.

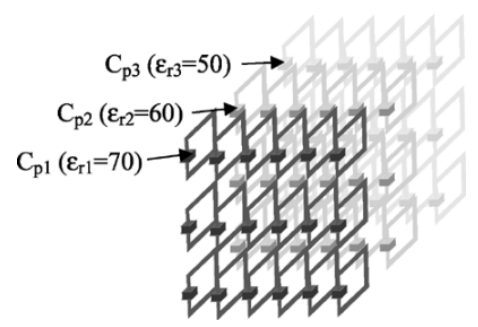

Fig. 3. Multiresonant three-layered ERC EBG material. The dimensions are the same as Fig. 1(a).

region cannot be controlled. The challenge is to modify the design so that a compact EBG architecture with a wide bandgap characteristic can be fabricated. To accomplish this, the idea of using multiple layers of embedded-circuits having different resonant frequencies is investigated.

The geometry of a three-layered ERC with different loop capacitors or resonant frequencies is depicted in Fig. 3. The FDTD is used to obtain the performance of each layer separately as well as the composite three-layered ERC, by observing the transmission coefficient. The results are shown in Fig. 4(a) and (b). Unfortunately, the three-layered ERC shows a multiresonant behavior instead of a simultaneous wide-band characteristic. The three-layered ERC is basically equivalent to three cascaded parallel $L C$ circuits having different resonant frequencies. In this 


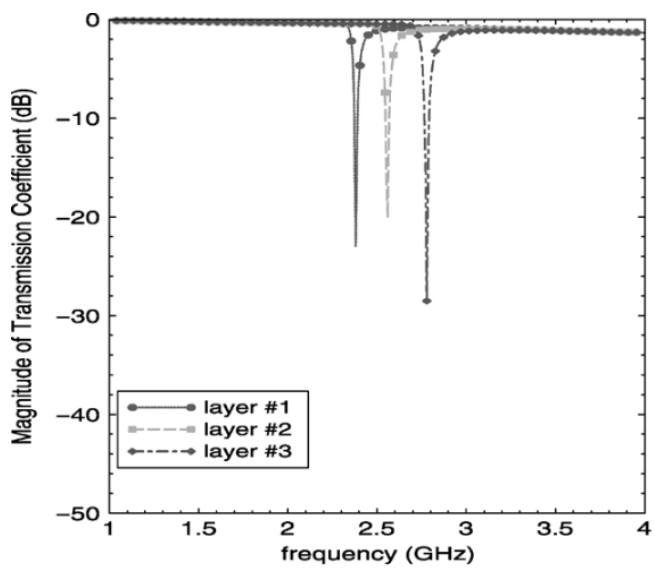

(a)

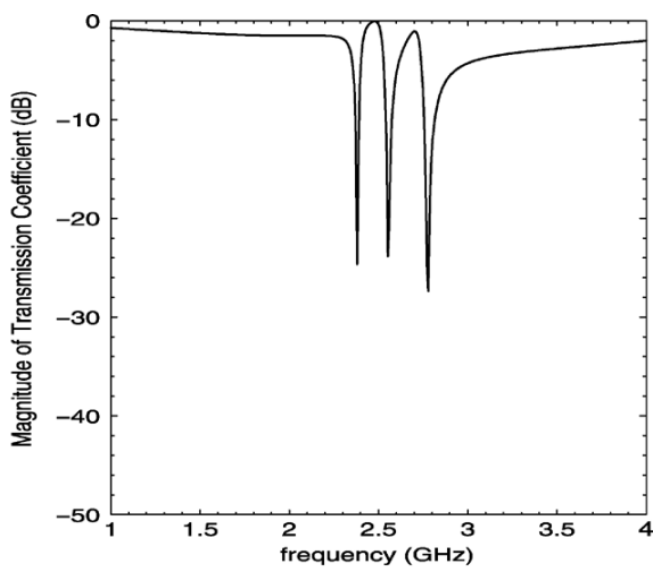

(b)

Fig. 4. Transmission coefficient for a plane wave propagating through the (a) each layer and (b) cascaded three layers of the three-resonant EBG medium designed in Fig. 3. Note the existence of transmission zeros between the poles of the multiresonant system.

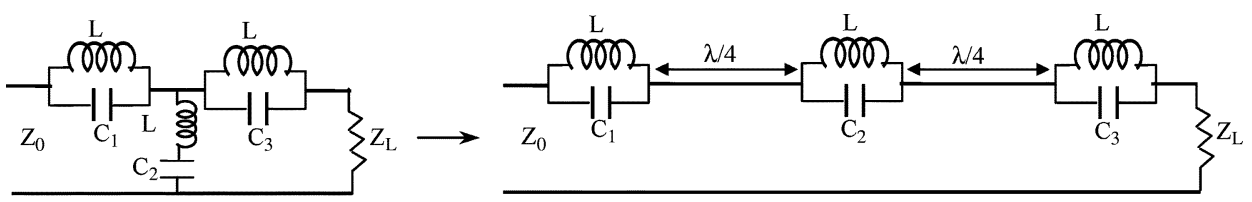

(a)

(b)

Fig. 5. (a) Circuit model representation for removing the transmission zeros occurring between the poles of parallel $L C$ circuits and providing a wide-band performance. (b) Its equivalent circuit model determined based on the $\lambda / 4$ impedance inverter concept.

network there is always a transmission zero between the adjacent poles. This disrupts the merging of the poles responses for achieving a wider bandgap. A standard approach in filter theory to overcome this problem is to introduce impedance inverters [15].

Consider the circuit model shown in Fig. 5(a) of a series $L C$ circuit with resonant frequency $f_{2}$ between two parallel $L C$ circuits with resonant frequencies $f_{1}$ and $f_{3}\left(f_{1}<f_{2}<f_{3}\right)$. In this design the series $L C$ circuit properly removes the transmission zero that occurs between the poles of the parallel $L C$ circuits and, therefore, a wide-band behavior can be obtained. The next step is to realize the circuit model in terms of an ERC EBG medium. Based on the concept of impedance inverters in filter theory, the circuit model in Fig. 5(a) is in fact equivalent to three parallel $L C$ circuits separated by $\lambda / 4$ [Fig. 5(b)]. Therefore, a three-layered ERC material constructed of layers with resonant frequencies $f_{1}, f_{2}$, and $f_{3}\left(f_{1}<f_{2}<f_{3}\right)$ that are located $\lambda / 4$ apart can produce the required wide-band performance. However, the $\lambda / 4$ separation increases the total dimension of the bandgap structure. To rectify this problem, $I$-shaped metallic strips with specific dimensions can be placed between the resonant circuits. The $I$-shaped metallic strips increase the permittivity of the host medium between the resonators [see (1a)] and thereby reduces the physical size of the $\lambda / 4$ sections.

Fig. 6 depicts the geometry of a wide-band compact ERC EBG structure. The transmission coefficients for a plane wave propagating through each layer and the whole structure are plotted in Fig. 7(a) and (b). A wide-band characteristic is illustrated for this novel design. A minimum $20-\mathrm{dB}$ isolation over $25 \%$ bandwidth is offered. Note that the unit cell of the structure has a very small size, about $0.01 \lambda_{0}$ in the $y$-direction

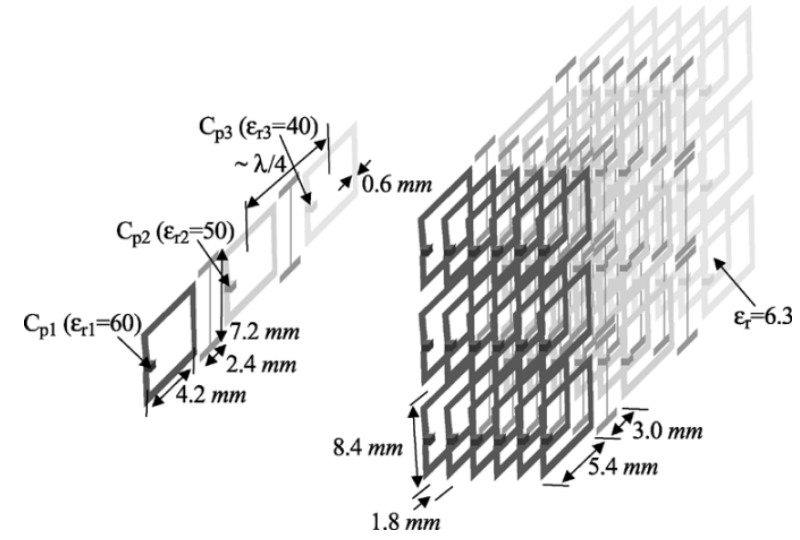

Fig. 6. Wide-band three-layered ERC EBG material. The $I$-shaped strips are inserted between the layers to increase the permittivity of host medium and reduce the required physical size for inverters.

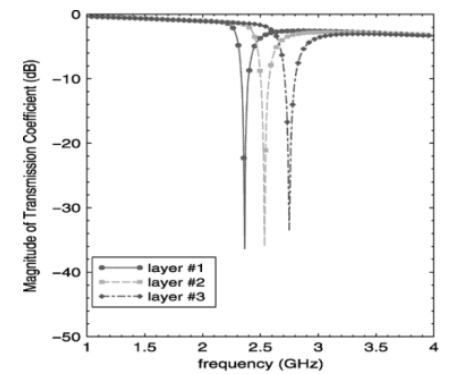

(a)

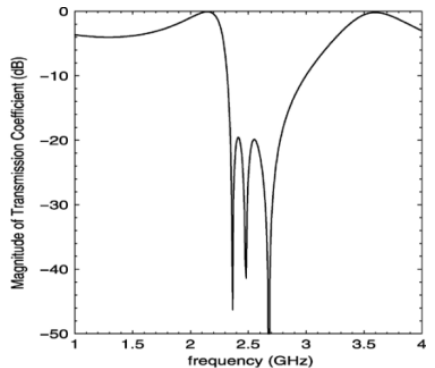

(b)
Fig. 7. Transmission coefficient for a plane wave propagating through the (a) each layer and (b) cascaded three layers of the wide-band EBG medium designed in Fig. 6. A wideband performance is demonstrated. 


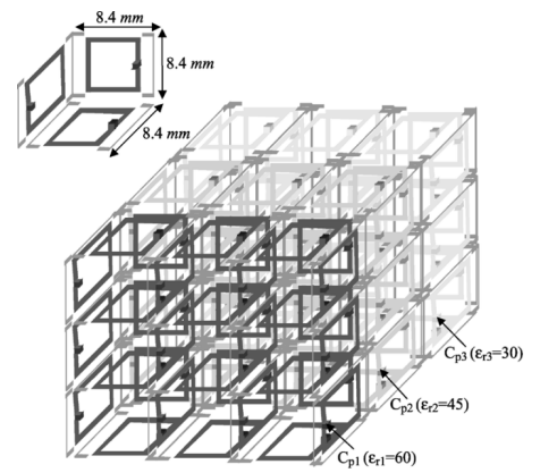

(a)

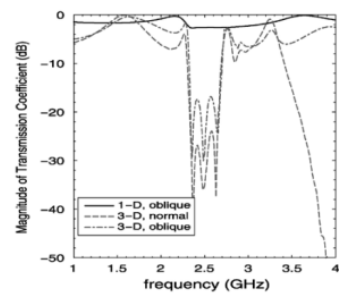

(b)
Fig. 8. Three-dimensional EBG with complete bandgap performance almost independent of incident angle and polarization state. (a) Geometry of ERC and I-shaped strips (their dimensions are the same as Fig. 6). (b) Transmission coefficient for the normal and oblique incidence $\left(\theta^{i}=90^{\circ}, \phi^{i}=150^{\circ}\right.$, and $\psi^{i}=40^{\circ}$ ) plane waves propagating through the structure. Also shown is the same response for the 1-D EBG (Fig. 6) at oblique incidence, which does not show the bandgap behavior.

and $0.06 \lambda_{0}$ in the $x$ - and $z$-directions ( $\lambda_{0}$ is the free space wavelength at the center frequency of bandgap region).

As discussed before, the bandgap region of an ERC medium is determined by the resonant behavior of the effective permeability. This phenomenon occurs only where the incident magnetic field has a component along the axes of the loops. To remove this anisotropic behavior and generate an isotropic EBG structure with a bandgap property independent of angle of incidence and polarization state, one needs to design a three-dimensional (3-D) periodic composite embedded-circuit material as shown in Fig. 8(a). The transmission coefficients for the normal incidence and an oblique incident plane wave with $\theta^{i}=90^{\circ}$, $\phi^{i}=150^{\circ}$, and a linear polarization specified by the angle $\psi^{i}=40^{\circ}$ (between the electric field and a reference direction $\left.\hat{\mathbf{k}}^{i} \times \hat{\mathbf{z}}\right)$ are plotted in Fig. 8(b). The lack of variation of the bandgap property to incidence angle and polarization is clearly demonstrated here. Shown in this figure is also the transmission coefficient for the designed one-dimensional (1-D) structure (Fig. 6) at the oblique incidence. As expected, the bandgap behavior of the 1-D structure is perturbed at the oblique incidence.

\section{CONCLUSION}

In this paper, the concept and design of a novel electromagnetic bandgap material with compact size and wide-band characteristic are presented. The EBG structure is constructed of resonant loop circuits periodically embedded in a dielectric host medium. The loop circuits have a tunable miniaturized size. Above the resonant frequency of ERC, a negative effective per- meability is generated which inhibits propagation of electromagnetic waves.

Cascading three layers of ERC with different resonant frequencies allows one to enhance the width of the bandgap region. The design characterization of a three-layered ERC EBG architecture with a small size and very wide bandgap behavior is demonstrated. $I$-shaped metallic strips are embedded in between the resonating layers in order to increase the dielectric of the medium between the layers and achieve a quarter wavelength inverter over a very short physical distance. A 3-D EBG with complete bandgap behavior, independent of incident angle and polarization state, is also demonstrated.

\section{REFERENCES}

[1] J. D. Joannopoulos, R. D. Meade, and J. N. Winn, Photonic Crystals: Molding the Flow of Light. Princeton, NJ: Princeton Univ. Press, 1995.

[2] E. Yablonovitch, "Inhibited spontaneous emission in solid-state physics and electronics," Phys. Rev. Lett., vol. 58, no. 20, pp. 2059-2062, May 1987.

[3] O. Painter, R. K. Lee, A. Scherer, A. Yariv, J. D. O’Brien, P. D. Dapkus, and I. Kim, "Two-dimensional photonic band-gap defect mode laser," Science, vol. 284, pp. 1819-1821, Jun. 1999.

[4] L. Shawn-Yu, E. Chow, V. Hietala, P. R. Villeneuve, and J. D. Joannopoulos, "Experimental demonstration of guiding and bending of electromagnetic waves in a photonic crystal," Science, vol. 282, pp. 274-276, Oct. 1998.

[5] Y. Rahmat-Samii and H. Mosallaei, "Electromagnetic band-gap structures: Classification, characterization, and applications," in Proc. 11th Int. Conf. Antennas and Propagation, Manchester, U.K., Apr. 17-20, 2001, pp. 560-564.

[6] H. Mosallaei and Y. Rahmat-Samii, "Periodic band-gap and effective dielectric materials in electromagnetics: characterization and applications in nanocavities and waveguides," IEEE Trans. Antennas Propagat., vol. 51, pp. 549-563, Mar. 2003.

[7] J. D. Shumpert, W. Chappell, and L. Katehi, "Parallel-plate mode reduction in conductor-backed slots using electromagnetic bandgap structures," IEEE Trans. Microwave Theory Tech., vol. 47, pp. 2099-2104, Nov. 1999.

[8] D. Sievenpiper, L. Zhang, R. F. J. Broas, N. G. Alexópoulos, and E. Yablonovitch, "High-impedance electromagnetic surfaces with a forbidden frequency band," IEEE Trans. Microwave Theory Tech., vol. 47, pp. 2059-2074, Nov. 1999.

[9] K. Sarabandi and H. Mosallaei, "Novel artificial embedded circuit meta-material for design of tunable electro-ferromagnetic permeability medium," in Proc. IEEE Int. Microwave Symp., vol. 3, Philadelphia, PA, Jun. 8-13, 2003, pp. 2061-2064.

[10] H. Mosallaei and Y. Rahmat-Samii, "Broadband characterization of complex periodic EBG structures: an FDTD/prony technique based on the split-field approach," Electromag. J., vol. 23, no. 2, pp. 135-151, Feb.-Mar. 2003.

[11] A. Taflove, Computational Electrodynamics: The Finite-Difference Time-Domain Method. Norwood, MA: Artech House, 1995.

[12] — Advances in Computational Electrodynamics: The Finite-Difference Time-Domain Method. Norwood, MA: Artech House, 1998.

[13] S. A. Schelkunoff and H. T. Friis, Antennas Theory and Practice. New York: Wiley, 1952.

[14] J. B. Pendry, A. J. Holden, D. J. Robbins, and W. J. Stewart, "Magnetism from conductors and enhanced nonlinear phenomena," IEEE Trans. Microwave Theory Tech., vol. 47, pp. 2075-2084, Nov. 1999.

[15] D. M. Pozar, Microwave Engineering. New York: Wiley, 1998. 